\title{
Optical Fiber Modes Resist Deformations
}

\author{
A machine-learning approach quickly characterizes an optical fiber, \\ identifying transmission channels that aren't affected by deformation.
}

\author{
By Rachel Berkowitz
}

M ultimode optical fibers, which can carry many simultaneous signals, offer a way to increase data rates beyond those of commonly used single-mode fibers. However, they are extremely sensitive to environmental disturbances that scramble the signals. Researchers have now developed a numerical approach to quickly estimate those disturbances and determine a set of signal channels in the fiber that are resistant to perturbations [1]. Using such robust channels would improve the performance of multimode fibers, which could boost transmission rates in communications networks and could lead to less invasive endoscopic probes for imaging bodily organs.

Light in a multimode fiber does not propagate down the center but rather in a zigzag path with reflections off the fiber's outer cylindrical shell. There are multiple zigzag paths, or modes, available in the fiber, each of which serves as a separate data channel. The modes may be distinguished by the spatial

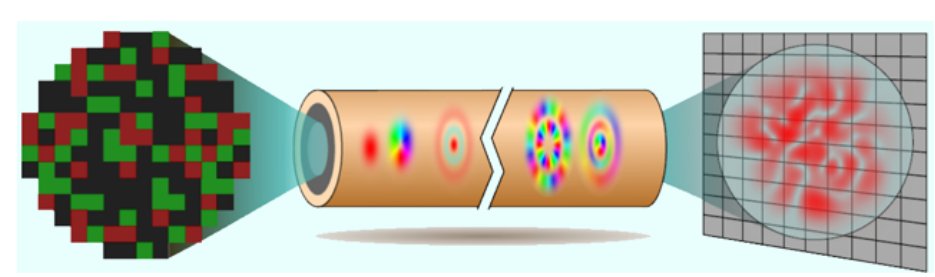

Fiber describer. Beams of spatially modulated light are sent through a multimode fiber. The recorded output light is fed into a machine-learning algorithm that determines how each mode, or data channel, in the fiber is scrambled by bending and other disturbances.

Credit: M. Matthès et al. [1] pattern of bright spots that each makes when shined directly onto a screen.

In a perfect, fixed-temperature fiber, a light signal injected in one mode emerges unchanged in the same mode at the end of the fiber. But fabrication defects, temperature changes, and fiber deformation, can cause a coupling between modes, which allows light to "leak" from one mode into other modes in a random fashion. Researchers characterize this mode-to-mode coupling with a mathematical entity called the transmission matrix. Obtaining a reliable transmission matrix requires very precise optical measurements that must be redone every time the fiber is bent or otherwise disturbed.

Now, borrowing tools from machine-learning frameworks, Sébastien Popoff of ESPCI Paris and his colleagues have developed an approach for rapidly measuring the transmission matrix and then using it to identify robust channels that are not very sensitive to changes in the fiber. The researchers first generated light beams with brightness variations across their profiles. They then injected these beams into a 30-cm length of fiber and imaged the light that emerged from the other end with a 1-kHz-frame-rate camera. After about $10 \mathrm{~s}$, they obtained a transmission matrix, which provides the relationship between the input light and output light at each spatial location, or "pixel," within the beam.

However, the pixel-to-pixel transmission matrix needs to be converted to the mode-to-mode transmission matrix in order to explore signal disruption. Such a conversion is made difficult by slight misalignments within the fiber and camera system. Rather than try to measure the misalignments, the researchers 


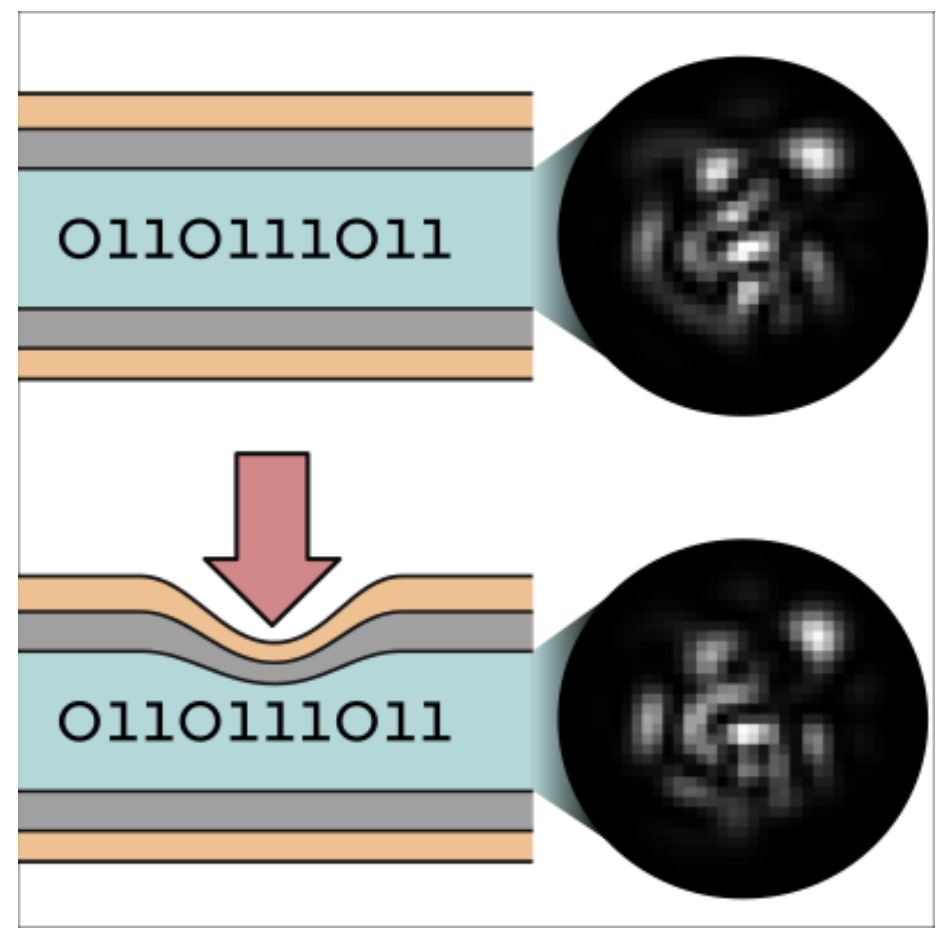

Data squeeze. Some modes of light injected into an optical fiber remain robust to deformations introduced by a pin pushing on the fiber jacket.

Credit: S. Popoff/ESPCI Paris

designed a numerical model that mimics the misalignments' effects. Through an optimization procedure, the model corrects for the misalignments and, within a few seconds, returns the desired mode-to-mode transmission matrix for a total of 110 modes. "We effectively shift the complexity from the experimental setup to the numerical optimization," says Popoff.

Having a method that can compute the transmission matrix in roughly $10 \mathrm{~s}$ allowed the researchers to study the effects of deformation. In a series of trials, they pressed on the fiber with a pin by a varying amount and repeated the transmission-matrix measurement each time. They determined that the deformation drastically affected the propagation of light in the fiber, but the impact was "localized" in the sense that light injected into one mode only coupled to certain other modes even for strong deformations.

The researchers then used their knowledge of the transmission matrix and its sensitivity to specific fiber deformations to choose a set of channels that remain insensitive to deformations. Selecting the most robust channels for use in multimode fibers could ensure that independent signals sent on each of those channels will not mix with others even when the configuration of the perturbation changes. "If I know my fiber will always be bent, and a channel is insensitive to that kind of deformation, then that channel will be ok even if the fiber bending changes over time," says Popoff.

Tomáš Čižmár, a fiber expert at the Leibniz Institute of Photonic Technology, Germany, says that the new method cuts down the time needed to determine the mode-to-mode transmission matrix. If the technique can be validated for a wider range of deformation types, "it could greatly stimulate numerous applications, including fiber-based endomicroscopy,” he says.

Rachel Berkowitz is a Corresponding Editor for Physics based in Vancouver, Canada.

\section{REFERENCES}

1. M. Matthès et al., "Learning and avoiding disorder in multimode fibers," Phys. Rev. X 11, 021060 (2021). 